\title{
Loss of Gucy1a3 Causes Poor Post-Stroke Recovery by Reducing Angiogenesis Via the HIF-1a/VEGFA Signaling Pathway in Mice
}

Man Luo

Guangxi Medical University First Affiliated Hospital

Dongcan Mo

Guangxi Medical University First Affiliated Hospital

LiuYu Liu

Guangxi Medical University First Affiliated Hospital

Jianli Li

Guangxi Medical University First Affiliated Hospital

Jing Lin

First Affiliated Hospital of Nanchang University

Jie Liang

Sun Yat-sen University First Affiliated Hospital

Fei Ye

Sun Yat-sen University First Affiliated Hospital

Xiaoju Wu

Guangxi Medical University First Affiliated Hospital

Xiaoling Li

Guangxi Medical University First Affiliated Hospital

Jiaoxing Li

Sun Yat-sen University First Affiliated Hospital

Wenli Sheng ( $\nabla$ shengwl@mail.sysu.edu.cn )

Sun Yat-sen University First Affiliated Hospital https://orcid.org/0000-0001-6867-371X

\section{Research Article}

Keywords: GUCY1A3, middle cerebral artery occlusion, ischemic stroke, angiogenesis, HIF-1a/VEGFA

Posted Date: January 6th, 2022

DOI: https://doi.org/10.21203/rs.3.rs-1220217/v1 
License: (c) (i) This work is licensed under a Creative Commons Attribution 4.0 International License. Read Full License 


\section{Abstract}

Ischemic stroke is a common and debilitating disease that can cause permanent neurological damage. Gucy1a3, which encodes the a1 subunit of soluble guanylyl cyclase, has been reported to be associated with functional recovery after ischemic stroke. However, the mechanism is still not well understood. In the present study, we investigated the effects of Gucy1a3 on (i) post-stroke recovery; (ii) vascular endothelial growth factor A (VEGFA) and hypoxia inducible factor 1 alpha (HIF-1a) expression; and (iii) angiogenesis after ischemic stroke.

A permanent middle cerebral artery occlusion (pMCAO) model was established using wild-type and Gucy1a3 knockout C57BL/6J male mice. Neurological deficits, infarct volume, microvascular density, and VEGFA and HIF-1a expression levels of mice were evaluated.

Our results suggest that loss of Gucy1a3 increased the infarct volume and aggravated neurological deficits after PMCAO. In addition, the Gucy1a3 knockout brains exhibited significantly lower microvessel densities and VEGFA and HIF-1a expression levels than the wild-type brains at 96 hours post-pMCAO.

The study shows that the expression of GUCY1A3 after ischemic stroke may play a substantial role in neurological function recovery and is related to angiogenesis in the peri-infarct region. The beneficial effects of GUCY1A3 might be mediated through the HIF-1a/VEGFA signaling pathway.

\section{Introduction}

Ischemic stroke is one of the leading causes of death and disability worldwide ${ }^{[1]}$. Although tissue plasminogen activator and endovascular thrombectomy have been established as effective therapies for ischemic stroke ${ }^{[2]}$, only a small population of patients benefits from these treatments because of the short therapeutic time window. Hence, there is an urgent need for effective therapeutic approaches, and the development of effective therapeutic strategies that can improve the recovery of blood flow and functional outcomes after stroke is highly desirable.

Angiogenesis refers to the formation of new blood vessels from pre-existing vasculature ${ }^{[3]}$. It occurs in the ischemic penumbra within hours after stroke and lasts for weeks. Cumulative evidence has indicated that post-ischemic angiogenesis is crucial for increasing the blood supply in the penumbral areas and improving the prognosis of stroke patients ${ }^{[4-6]}$. Therefore, developing new therapies to augment angiogenesis is of utmost importance for the treatment of ischemic stroke.

The Gucy1a3 gene encodes the a1 subunit of soluble guanylate cyclase (sGC), which is the most abundant SGC isoform in the vascular system. SGC is a heterodimeric enzyme composed of one $a$ and one $\beta$ subunit. The $\beta$ subunit also associates with the $a 2$ subunit, which is expressed at a lower level. sGC is the major receptor for nitric oxide (NO). NO binds the heme iron of SGC to induce cyclic guanosine monophosphate (cGMP) production, which then activates the cGMP-dependent protein kinase pathway [7]. It is widely accepted that NO plays an important role in angiogenesis ${ }^{[8,9]}$. Previous studies have 
reported that administration of an NO donor to rats after stroke significantly enlarged vascular perimeters and increased the numbers of proliferating cerebral endothelial cells and newly generated vessels in the ischemic boundary regions ${ }^{[10]}$. Mice lacking endothelial nitric oxide synthase showed severe impairment of angiogenesis in response to limb ischemia, and administration of an NO donor accelerated angiogenesis ${ }^{[11]}$. In addition, both in vitro and in vivo experiments have demonstrated that NO can promote endothelial cell proliferation and migration and tip cell sprouting, all of which are important steps in angiogenesis ${ }^{[12-14]}$.

Different mutations or polymorphisms in the Gucy1a3 gene are associated with various vascular phenotypes, including ischemic stroke ${ }^{[15]}$, moyamoya cerebral arteriopathy ${ }^{[16]}$, coronary artery disease ${ }^{[17]}$, and pulmonary artery hypertension ${ }^{[18]}$. A recent study found that homozygous Gucy 1 a3 risk allele (rs7692387) carriers were at increased risk of ischemic events early after coronary stenting ${ }^{[19]}$. In addition, a previous study reported that SGC a1 subunit deficiency in a mouse model of transient middle cerebral artery occlusion (MCAO) significantly reduced cerebral blood flow, enlarged infarct volumes, and aggravated neurological deficits, suggesting that the SGC a1 subunit is protective in ischemic stroke ${ }^{[20]}$. However, whether suppression of GUCY1A3 function can inhibit vascular regeneration in the brain after stroke is unknown.

In this study, we aimed to examine the role of GUCY1A3 in the regulation of angiogenesis in the ischemic brain. A Gucy1a3 knockout (Gucy1a3-KO) mouse model was generated to investigate the effects and potential molecular mechanisms of GUCY1A3 in cerebral ischemia-induced angiogenesis.

\section{Materials And Methods}

\section{Animals}

Male C57BL/6J mice (8- to 10-week-old, 21-25g) were used. All animal experiments were performed in accordance with approved protocols of the Sun Yat-sen University Animal Care and Use Committee and were in line with the Guide for the Care and Use of Laboratory Animals of the National Institute of Health in China. Mice were maintained on a 12-hour light/dark cycle at $22 \pm 2^{\circ} \mathrm{C}$ and allowed access to food and water ad libitum before the experiments. Gucy1a3 knockout (KO) mouse model was established by the CRISPR/Cas 9 system, which was described in detail in the Supplementary Materials.

\section{Permanent Middle Cerebral Artery Occlusion (pMCAO) for Stroke Model}

The left middle cerebral artery was permanently occluded using the method described previously [21]. Briefly, mice were anaesthetized with chloral hydrate $(200 \mathrm{mg} / \mathrm{kg}$, intraperitoneal injection), and their fur and skin were disinfected. The left common carotid artery, bifurcation of the internal common carotid artery (ICA), and external common carotid artery (ECA) were carefully dissected. A 5-0 nylon filament, with its tip rounded by heating near a flame, was inserted through the ECA stump and gently advanced (about 
$10 \mathrm{~mm}$ ) into the left internal carotid artery until it blocked the origin of the MCA. The wound and skin were was closed and the filament left in place. The body temperature of the mice was maintained $37.0 \pm 0.5^{\circ} \mathrm{C}$ with a heating pad during surgery.

\section{Neurological Scoring}

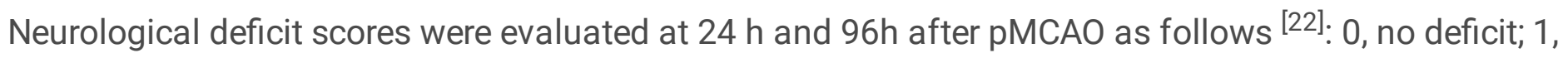
flexion of contralateral forelimb upon lifting the whole animal by the tail; 2 , circling to the contralateral side; 3 , falling to the contralateral side; and 4 , no spontaneous motor activity.

\section{Infarct volume}

The cerebral infarct volume was determined by 2,3,5-triphenyltetrazolium chloride (TTC) staining. The brains of mouse with 96 hours after pMCAO surgery were taken directly after anesthesia, and they were frozen quickly in $-20^{\circ} \mathrm{C}$ refrigerator for about $20 \mathrm{~min}$. Then the brain was taken out and placed on a special brain trough for 5 consecutive slices with a thickness of $2 \mathrm{~mm}$. The slices were placed in $2 \%$ TTC (Sigma Aldrich, MO, USA), soaked for $30 \mathrm{~min}$ at $37^{\circ} \mathrm{C}$ with shaded light, and finally fixed with $4 \%$ paraformaldehyde. After the above staining, red represents normal brain tissue, and white represents infarcted brain tissue. The sections were arranged in sequence, labeled, scaled and photographed, and the volume of cerebral infarction was calculated by ImageJ analyzer software (National Institutes of Health, USA). The infarct area of each slice was calculated, and then the total infarct volume was calculated by multiplying the infarct area of each slice by the thickness $(2 \mathrm{~mm})$.

\section{Immunofluorescence}

Ninety-six hours after the PMCAO procedure, the brains were collected quickly and post-fixed in $4 \%$ paraformaldehyde overnight. Serial coronal sections of the mouse brain $(8 \mu \mathrm{m}$ thick) were cut using a cryostat. Sections were incubated with $3 \% \mathrm{BSA}$ for $30 \mathrm{~min}$ at $37^{\circ} \mathrm{C}$ and then immune-stained with primary antibodies against CD31 (endothelial cell marker, 1:1000, Abcam, MA, USA) and VEGFA antibody (1:250, Abcam, MA, USA). Following overnight incubation, the sections were washed and subsequently incubated with secondary antibodies Cy3-conjugated donkey anti-mouse IgG and Alexa 488 IgG (Cys for red and Alexa 488 for green, 1:1000, Invitrogen, CA, USA) for $1 \mathrm{~h}$ at $37^{\circ} \mathrm{C}$. Images obtained using a fluorescence microscope (Leica-LCS-SP8-STED, Germany). And images area and intensity were measured in ImageJ.

\section{Microvascular density (MVD)}

CD31 was used to mark endothelial cell and evaluate the MVD as previously described ${ }^{[23]}$. Briefly, the $\mathrm{CD} 31^{+}$cells apart from the vessel, no matter alone or in groups, would be regarded as a microvessel and should be counted. 6 slices of a group were counted and the number of microvessels was counted in five sights of $400 \times$ field (i.e., $40 \times$ objective lens and 10xocular lens) for calculating the MVD (number of microvessels per $\mathrm{mm}^{2}$ ). Counting was done by two independent observers and the results are expressed as means \pm SD.

\section{Western Blot Analysis}


The brain tissue from the peri-infarct region was collected at 96 hours after stroke. Tissue samples were homogenized in RIPA buffer (Beyotime Biotechnology, Shanghai, China) containing PMSF (Beyotime Biotechnology, Shanghai, China) and crushed by an ultrasonic comminution machine. After a 30min centrifugation at $12000 \mathrm{rpm}$, the supernatant (the total protein) was harvested. After that, the proteins were separated by $10 \%$ sodium dodecyl sulfate-polyacrylamide gel electrophoresis and the proteins were transferred onto the PVDF membranes. Subsequent to blocking with QuickBlock blocking buffer for 30 min at $25^{\circ} \mathrm{C}$ followed by three washes for 10 min with western wash buffer, the membranes were incubated overnight at $4^{\circ} \mathrm{C}$ with SGC a1 antibodies (1:3000, Abcam, MA, USA), HIF1 a mouse monoclonal antibody (1:3000, Affinity, OH, USA), Vegfa monoclonal antibody (1:2000, Invitrogen, CA, USA) and GAPDH antibody (1:5000, Affınity, OH, USA). Following three washes for 10 min with TBST, the samples were incubated with the secondary antibody, goat anti-rabbit immunoglobulin G (1:3000, Abcam, MA, USA) and membrane was finally visualized using the chemiluminescence detection system (Thermo Scientific, IL, USA).

\section{Statistics}

Statistical analyses were performed using SPSS software (SPSS Inc., IL, USA). The data were given as mean \pm standard deviation. Comparisons between two groups were analyzed by Student's $t$ tests. Data comparisons between multiple groups were analyzed by one-way ANOVAs with Tukey's post hoc tests. P values $<0.05$ were considered statistically significant.

\section{Results}

\subsection{Generation of Gucy1a3-KO mice}

Gucy1a3-modified mice were generated via co-microinjection of Gucy1a3 single-guide RNA (targeting exon 5, Fig. 1a) and Cas9 mRNA into the cytoplasm of C57BL/6J zygotes. Gucy1a3-modified mice were mated with wild-type (WT) mice to obtain mutant mice heterozygous for the Gucy1a3 mutation. Then, Gucy1a3 heterozygous mutant mice were intercrossed to produce homozygous Gucy1a3 global knockout mice. Homozygous Gucy1a3-KO mice were born at a normal Mendelian ratio and did not show apparent alterations or a reduced life expectancy. All mice were genotyped by sequencing for the CRISPR/Cas9induced mutation in the exon 5 of the Gucy1a3 locus, and a 13-base pair deletion was found in Gucy1a3$\mathrm{KO}$ mice (Fig. 1C). Western blot analysis was performed to confirm the loss of the sGC a1 subunit. As shown in Fig. 1d, 1e, the protein was completely absent in the brains of Gucy1a3-KO mice, which confirmed at the protein level that Gucy1a3-KO mice were successfully generated.

\subsection{Loss of Gucy1a3 increases the infarction volume and neurological deficits after ischemic stroke}

To evaluate the protective effects of GUCY1A3 against stroke, we measured the infarct volumes and neurological deficit scores in a mouse model of pMCAO. The infarct volumes were evaluated by TTC 
staining 96 hours after the pMCAO operation, and the neurological deficit scores were evaluated at 24 and 96 hours after successful modeling using the Longa score ( $0-4$ points) method. The infarct volume 96 hours after PMCAO, represented as the percentage of hemispheric volume, was significantly larger in Gucy1a3-KO mice than in WT mice (23.8 $\pm 6.1 \%$ vs. $17.0 \pm 5.1 \%, P<0.05$, Fig. $2 \mathrm{a}, 2 \mathrm{~b})$. Furthermore, there was no significant difference in neurological deficit scores between WT and Gucy1a3-KO mice 24 hours after pMCAO, but neurological deficits were significantly greater in Gucy1a3-KO mice at 96 hours after the operation ( $P<0.05$, Fig. $2 \mathrm{C})$.

\subsection{Loss of Gucy1a3 impairs angiogenesis after ischemic stroke}

Angiogenesis is a prerequisite for recovery from ischemic stroke; hence, we determined whether GUCY1A3 could promote angiogenesis after experimental stroke. Microvessels were assessed by calculating the areas of cells that expressed the endothelial marker CD31 in the perifocal striatum region. CD31 immunofluorescence analysis showed a much higher microvessel density in WT mice than in Gucy1a3KO mice at 96 hours post-MCAO (Fig. 3a, 3b). This result suggests that Gucy1a3 deletion impaired angiogenesis after ischemic stroke; therefore, Gucy1a3 might promote angiogenesis after focal ischemia.

\subsection{Loss of Gucy1a3 decreases angiogenic factor expression in ischemic stroke}

Angiogenesis is largely driven by vascular endothelial growth factor A (VEGFA) production in response to hypoxia. To verify angiogenic dysfunction in Gucy1a3-KO mice, we detected VEGFA expression in the perifocal striatum of Gucy1a3-KO mice using immunofluorescence and western blotting. Double immunofluorescence staining of CD31 and VEGFA showed that VEGFA was primarily localized in endothelial cells. The immune expression of VEGFA was decreased in the infarcted striatum of Gucy1a3KO mice compared with that of WT mice (Fig. 4a, 4b). Furthermore, the VEGFA protein level of ischemic mice at 96 hours post-pMCAO was significantly lower in the Gucy1a3-KO group, as measured by western blotting (Fig. 4c, 4e).

Hypoxia inducible factor 1a (HIF-1a) is a key regulator of VEGFA production in ischemic tissues. Inhibition of the HIF-1a signaling blocks angiogenesis in various diseases. To assess the effects of Gucy1a3-KO on HIF-1a, we analyzed the HIF-1a expression levels in the ipsilateral striatum homogenate obtained from the KO and WT groups at 96 hours post-pMCAO using western blotting. The results showed that Gucy1a3-KO decreased the HIF-1a protein level significantly (Fig. 4c, 4d).

\section{Discussion}

Ischemic stroke is a common clinical disease that is associated with high levels of disability and mortality and seriously threatens human life and health. In recent years, Gucy 1a3, which encodes the a1 subunit of SGC, has been proposed to be tightly correlated with cerebrovascular diseases and coronary 
artery disease ${ }^{[15-17,24]}$. However, only a few studies have examined the effects of GUCY1A3 on ischemic stroke, and the mechanisms underlying these effects remain unclear. In the present study, Gucy1a3-KO mice were used to investigate the effects of GUCY1A3 on ischemic stroke. Our results indicated that loss of Gucy1a3 increased the infarct volume and aggravated neurological deficits after pMCAO. Moreover, Gucy1a3-KO brains exhibited significantly lower microvessel densities than WT brains at 96 hours postpMCAO, suggesting that Gucy1a3 deletion suppressed angiogenesis after ischemic stroke. Further analysis confirmed that loss of Gucy1a3 decreased VEGFA and HIF-1a expression levels, which may contribute to the decrease in angiogenesis in Gucy1a3-KO mice after pMCAO.

The impact of genetic variations in the Gucy1a3 gene on ischemic cardio-cerebrovascular diseases has been highlighted by previous studies. Variants of the Gucy1a3 gene are enriched in patients suffering from ischemic stroke and coronary artery disease ${ }^{[15,24,25]}$. A previous study has also shown that the infarct size was larger and the neurologic outcome was worse in $\mathrm{sGCa} 1^{-/-}$mice than in WT mice subjected to 1 hour of MCAO and 23 hours of reperfusion, but the infarct volumes and neurological deficits were similar after 24 hours of permanent occlusion in both genotypes ${ }^{[20]}$. That study further revealed that impaired vascular relaxation may underlie the more marked reperfusion deficit observed in $\mathrm{sGCa}^{-/-}$mice than in WT mice after middle cerebral artery reperfusion ${ }^{[20]}$. Interestingly, our results showed no significant difference in neurological deficit scores between WT and Gucy1a3-KO mice 24 hours after PMCAO, but neurological deficits and the infarct volume were significantly greater in Gucy1a3$\mathrm{KO}$ mice at 96 hours after PMCAO. However, temporal changes in the effects of sGCa1 deficiency on infarct volume and neurological deficits following PMCAO cannot be fully explained by impaired vascular relaxation.

Previous studies have confirmed that angiogenesis is correlated with improved functional recovery and prolonged survival after ischemic stroke ${ }^{[6,26]}$. Krupinski et al. ${ }^{[6]}$ reported that angiogenic activity occurred in ischemic stroke patients 3-4 days after stroke. In rodent stroke models, endothelial cells in the periinfarct brain tissue start to proliferate as early as $12-24$ hours following ischemic stroke; accordingly, vessel density significantly increases in the peri-infarct region 3 days after ischemia ${ }^{[27,28]}$. In our study, after 4 days ( 96 hours) of pMCAO, the microvessel density was lower in Gucy1a3-KO mice than in WT mice. A larger infarct volume and worse functional outcome were also observed, which might have resulted from the decreased angiogenic activity in the peri-infarct region.

VEGFA is the most important angiogenesis-promoting factor and plays an important role in angiogenesis after focal cerebral ischemia ${ }^{[29]}$. As a key oxygen concentration-dependent transcription factor, HIF-1a can regulate multiple target genes, including VEGFA, and thereby modulate angiogenesis after ischemia ${ }^{[30]}$. As mentioned previously, SGC is the major cytosolic receptor for NO, catalyzing the conversion of guanosine-5'-triphosphate to cGMP. NO and hypoxia were reported to upregulate the VEGFA gene by enhancing HIF-1 activity ${ }^{[31,32]}$. We further examined whether the decreased angiogenesis in the Gucy1a3$\mathrm{KO}$ ischemic brain could be explained by the decrease in HIF-1a/VEGFA expression. The results showed that, compared with the WT group, the VEGFA and HIF-1a protein levels were significantly decreased at 96 
hours after ischemia in Gucy1a3-KO mice, suggesting that, mechanistically, the HIF-1a/VEGFA-dependent signaling may mediate the effects of GUCY1A3 on angiogenesis.

Several limitations of this study should be considered. First, in the current study, only silencing experiments were performed. In the next set of experiments, overexpression of the Gucy1a3 gene should be employed to investigate the impact of GUCY1A3 on angiogenesis and cerebral injury after ischemic stroke. Second, a previously published paper suggested that hypertension and the responsiveness to NO in sGCa1 knockout mice are gender-specific ${ }^{[33]}$. Therefore, female Gucy1a3-KO mice should be investigated in the future. Third, compensatory mechanisms in the Gucy1a3-KO model, including a compensatory increase in GUCY1A2 expression in Gucy1a3-KO mice, were not evaluated in the present study. Nevertheless, other studies have confirmed that loss of one of the SGC isoforms was not compensated by an upregulation of the remaining one, indicating that deletion of the SGCa1 subunit is not compensated by upregulation of the other form ${ }^{[33,34]}$.

In conclusion, we demonstrated that GUCY1A3 expression after ischemic stroke may play a significant role in neurological function recovery, which is related to angiogenesis in the peri-infarct region. The beneficial effects of GUCY1A3 might be mediated by the HIF-1a/VEGFA signaling pathway. This raises the possibility of targeting Gucy $1 a 3$ as a reasonable and novel therapeutic strategy for ischemic stroke.

\section{Declarations}

\section{Acknowledgements}

We thank the Guangdong Engineering \& Technology Research Center for Disease-Model Animals.

\section{Declaration of conflicting interests}

The authors have no relevant financial or non-financial interests to disclose.

\section{Funding}

This work was supported by the National Natural Science Foundation of China (Grant no. 81471180, 81671132 and 82071286), the National Natural Science Foundation of China for Young Scientists Fund (Grant no.81701142 and 82101405), the Natural Science Foundation of Guangxi Province (Grant no.2015GXNSFAA139171 and 2020GXNSFAA259053), and Guangxi Scholarship Fund of Guangxi Education Department.

\section{Author Contributions}

All authors contributed to the study conception and design. Material preparation, data collection and analysis were performed by Man Luo, Dongcan Mo, LiuYu Liu, Jianli Li, Jing Lin, Jie Liang, Fei Ye, Xiaoju 
Wu, Xiaoling Li, Jiaoxing Li. The first draft of the manuscript was written by Man Luo and all authors commented on previous versions of the manuscript. Wenli Sheng contributed to design, analysis and interpretation of data and editing and approving the final manuscript. All authors read and approved the final manuscript.

\section{Data Availability}

The datasets generated or analysed during the current study are not publicly available due to the co-first author Dongcan Mo is preparing his master's thesis, this research is a part of the master's thesis but is available from the corresponding author on reasonable request.

\section{Ethics approval}

All animal experiments were performed in accordance with approved protocols of the Sun Yat-sen University Animal Care and Use Committee and were in line with the Guide for the Care and Use of Laboratory Animals of the National Institute of Health in China.

\section{Consent for Publication}

Not applicable.

\section{Consent to Participate}

Not applicable.

\section{References}

1. Pallesen LP, Barlinn K, Puetz V (Role of decompressive craniectomy in ischemic stroke. Front Neurol 9:1119.

2. Nogueira RG, Jadhav AP, Haussen DC (Thrombectomy 6 to hours after stroke with a mismatch between deficit and infarct. N Engl J Med 378:11-21.

3. Azad T, Ghahremani M, Yang X (The role of YAP and TAZ in angiogenesis and vascular mimicry. Cells 8:407.

4. Beck H, Plate KH (Angiogenesis after cerebral ischemia. Acta Neuropathol 117:481-496.

5. Zhang ZG, Chopp M (Neurorestorative therapies for stroke: underlying mechanisms and translation to the clinic. Lancet Neurol 8:491-500.

6. Krupinski J, Kaluza J, Kumar P, Kumar S, Wang JM (Role of angiogenesis in patients with cerebral ischemic stroke. Stroke 25:1794-1798.

7. Zhang Y, Janssens SP, Wingler K, Schmidt HH, Moens AL (Modulating endothelial nitric oxide synthase: a new cardiovascular therapeutic strategy. Am J Physiol Heart Circ Physiol 301:H634-646.

8. Ziche M, Morbidelli L (Nitric oxide and angiogenesis. J Neurooncol 50:139-148. 
9. Cooke JP, Losordo DW (Nitric oxide and angiogenesis. Circulation 105:2133-2135.

10. Zhang $R$, Wang $L$, Zhang $L$ (Nitric oxide enhances angiogenesis via the synthesis of vascular endothelial growth factor and cGMP after stroke in the rat. Circ Res 92:308-313.

11. Murohara T, Asahara T, Silver M, Bauters C, Masuda H, Kalka C et al (Nitric oxide synthase modulates angiogenesis in response to tissue ischemia. J Clin Invest 101:2567-2578.

12. Ziche M, Parenti A, Ledda F (Nitric oxide promotes proliferation and plasminogen activator production by coronary venular endothelium through endogenous bFGF. J Circ Res 80:845-852.

13. Eller-Borges R, Batista WL, da Costa PE, Tokikawa R, Curcio MF, Strumillo ST et al (Ras, Racand phosphatidylinositol-3-kinase (PI3K) signaling in nitric oxide induced endothelial cell migration. Nitric Oxide 47:40-51.

14. Mani KP, Giriraj S, David RS, Naga G, Abaya MS, Vivek J et al (Tipping off endothelial tubes: nitric oxide drives tip cells. Angiogenesis 18:175-189.

15. Li JL, Liu LY, Jiang DD, Jiang YY, Zhou GQ, Mo DC et al (Associations between GUCY1A3 genetic polymorphisms and large artery atherosclerotic stroke risk in Chinese Han population: a case-control study. Lipids Health Dis 18:233.

16. Wallace S, Guo DC, Regalado E, Mellor-Crummey L, Bamshad M, Nickerson DA et al (Disrupted nitric oxide signaling due to GUCY1A3 mutations increases risk for moyamoya disease, achalasia and hypertension. Clin Genet 90:351-960.

17. Erdmann J, Stark K, Esslinger UB, Rumpf PM, Koesling D, de Wit C et al (Dysfunctional nitric oxide signalling increases risk of myocardial infarction. Nature 504:432-436.

18. Wilkins MR, Aldashev AA, Wharton J, Rhodes CJ, Vandrovcova J, Kasperaviciute D et al (a1-Avariant in GUCY1A3 as a candidate conferring protection from pulmonary hypertension among Kyrgyz highlanders. Circ Cardiovasc Genet 7:920-929.

19. Kessler T, Wolf B, Eriksson N, Kofink D, Mahmoodi BK (Association of the coronary artery disease risk gene GUCY1A3 with ischaemic events after coronary intervention. Cardiovasc Res 115:1512-1518.

20. Atochin DN, Yuzawa I, Li Q, Rauwerdink KM, Malhotra R, Chang J et al (Soluble guanylate cyclase alpha1beta1 limits stroke size and attenuates neurological injury. Stroke 41:1815-1819.

21. Tsuchiya D, Hong S, Matsumori Y, Shiina H, Kayama T, Swanson RA et al (Overexpression of rat heat shock protein is associated with reduction of early mitochondrial cytochrome $\mathrm{C}$ release and subsequent DNA fragmentation after permanent focal ischemia. J Cereb Blood Flow Metab 23:718727.

22. Longa EZ, Weinstein PR, Carlson S, Cummins R (Reversible middle cerebral artery occlusion without craniectomy in rats. Stroke 20:84-91.

23. Weidner N, Semple JP, Welch WR, Folkman J (Tumor angiogenesis and metastasis-correlation in invasive breast carcinoma. N Engl J Med 324:1-8.

24. Kessler T, Wobst J, Wolf B, Eckhold J, Vilne B, Hollstein R, et al (Functional characterization of the GUCY1A3 coronary artery disease risk locus. Circulation 136:476-489. 
25. Wobst J, von Ameln S, Wolf B, Wierer M, Dang TA, Sager HB et al (Stimulators of the soluble guanylyl cyclase: promising functional insights from rare coding atherosclerosis-related GUCY1A3 variants. Basic Res Cardiol 111:51.

26. Arenillas JF, Sobrino T, Castillo J, Davalos A (The role of angiogenesis in damage and recovery from ischemic stroke. Curr Treat Options Cardiovasc Med 9:205-212.

27. Vallon M, Chang J, Zhang H, Kuo CJ (Developmental and pathological angiogenesis in the central nervous system. Cell Mol Life Sci 71:3489-3506.

28. Hayashi T, Noshita N, Sugawara T, Chan PH (Temporal profile of angiogenesis and expression of related genes in the brain after ischemia. J Cereb Blood Flow Metab 23:166-180.

29. Hermann DM, Zechariah A (Implications of vascular endothelial growth factor for postischemic neurovascular remodeling. J Cereb Blood Flow Metab 29:1620-1643.

30. Tirpe AA, Gulei D, Ciortea SM, Crivii C, Berindan-Neagoe I (Hypoxia: Overview on hypoxia-mediated mechanisms with a focus on the role of HIF genes. Int J Mol Sci 20:6140.

31. Kimura $H$, Esumi $H$ (Reciprocal regulation between nitric oxide and vascular endothelial growth factor in angiogenesis. Acta Biochim Pol 50:49-59.

32. Kimura H, Weisz A, Kurashima Y, Hashimoto K, Ogura T, D'Acquisto F et al (Hypoxia response element of the human vascular endothelial growth factor gene mediates transcriptional regulation by nitric oxide: control of hypoxia-inducible factor- 1 activity by nitric oxide. Blood 95:189-197.

33. Emmanuel SB, Patrick S, Pieter V, Michael JR, Elke R, Fumito I et al (Gender-specific hypertension and responsiveness to nitric oxide in SGCalpha1 knockout mice. Cardiovasc Res 79:179-186.

34. Evanthia M, Andreas F, Oliver D, Michael R, Doris K (Spare guanylyl cyclase NO receptors ensure high NO sensitivity in the vascular system. J Clin Invest 116:1731-1737.

\section{Figures}


a

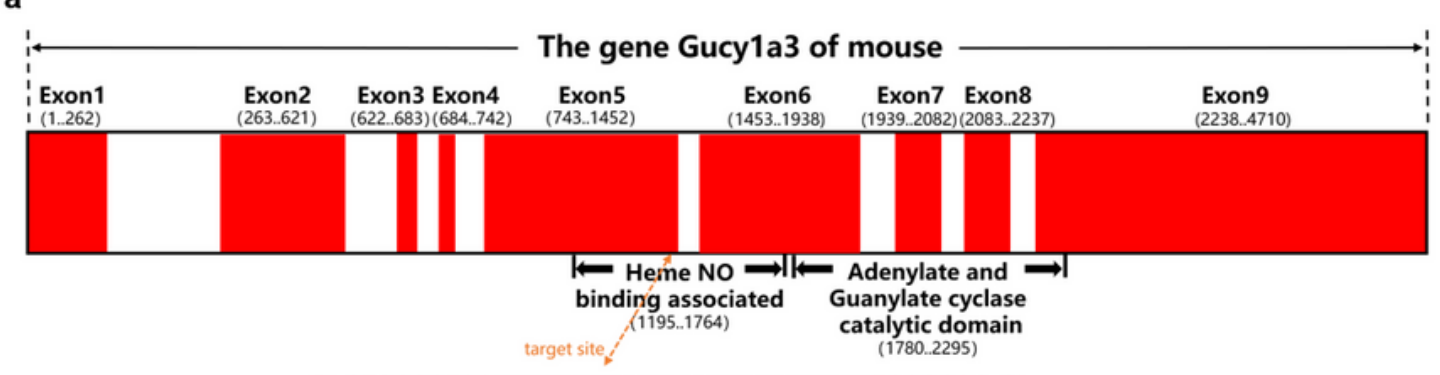

5'-ATGGGATAACTCGGTGAAGAAATCGTCAAGGGTAAGGAA-3'

b

ATAACTCGGTGAAGAAATCGTCAAGGGTAAGGAAAATGTAACGCGGATTCAAA.

WT

C

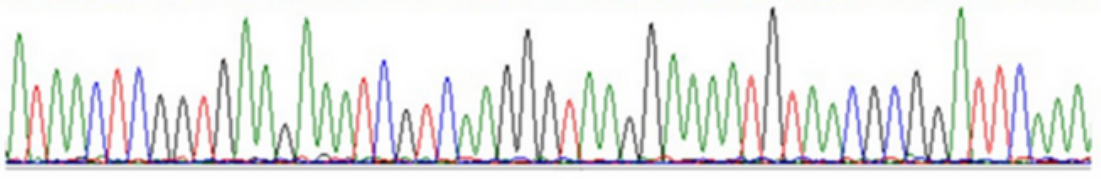

$\downarrow^{\text {-TCGTCAAGgGtaA }}$

ATAACTCGGTGAAGAAAGGAAAT GTAACG G GATCAAAATAAAACCAAT TG

KO

$-13 b p$ mutant
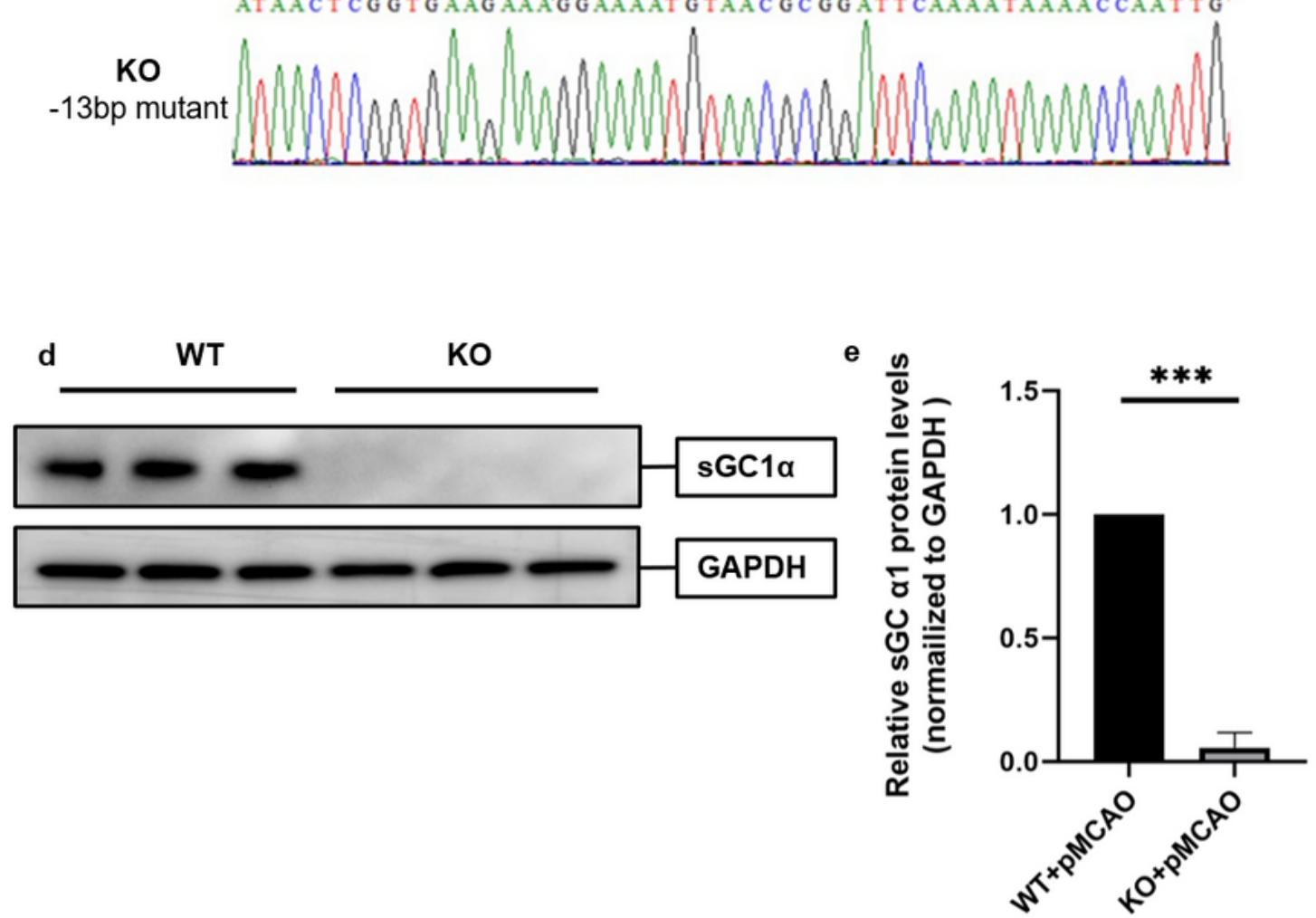

Figure 1

Sequence alignment and western blot analysis of Gucy1a3-KO mice

(a) Mouse Gucy1a3 gene structure and single-guide RNA design. A diagram of the mouse Gucy1a3 locus with nine exons is shown. The single-guide RNA guiding sequence and protospacer adjacent motif at the target site are shown in red and black, respectively. (b) The Gucy1a3 gene sequencing result of a WT 
mouse. (c) The Gucy1a3 gene sequencing result of a homozygous Gucy1a3-KO mouse. The black arrow and base sequence represent the 13-base pair deletion. (d) The representative bands of sGC a1 and GAPDH detected by Western blotting in WT and KO mice. (e) Quantitative results of the SGC a1 to GAPDH bands. Data are expressed as the means $\pm S D$ ( $n=3$ per group). WT, wild-type; KO, knockout. ${ }^{* \star *} P<0.001$.

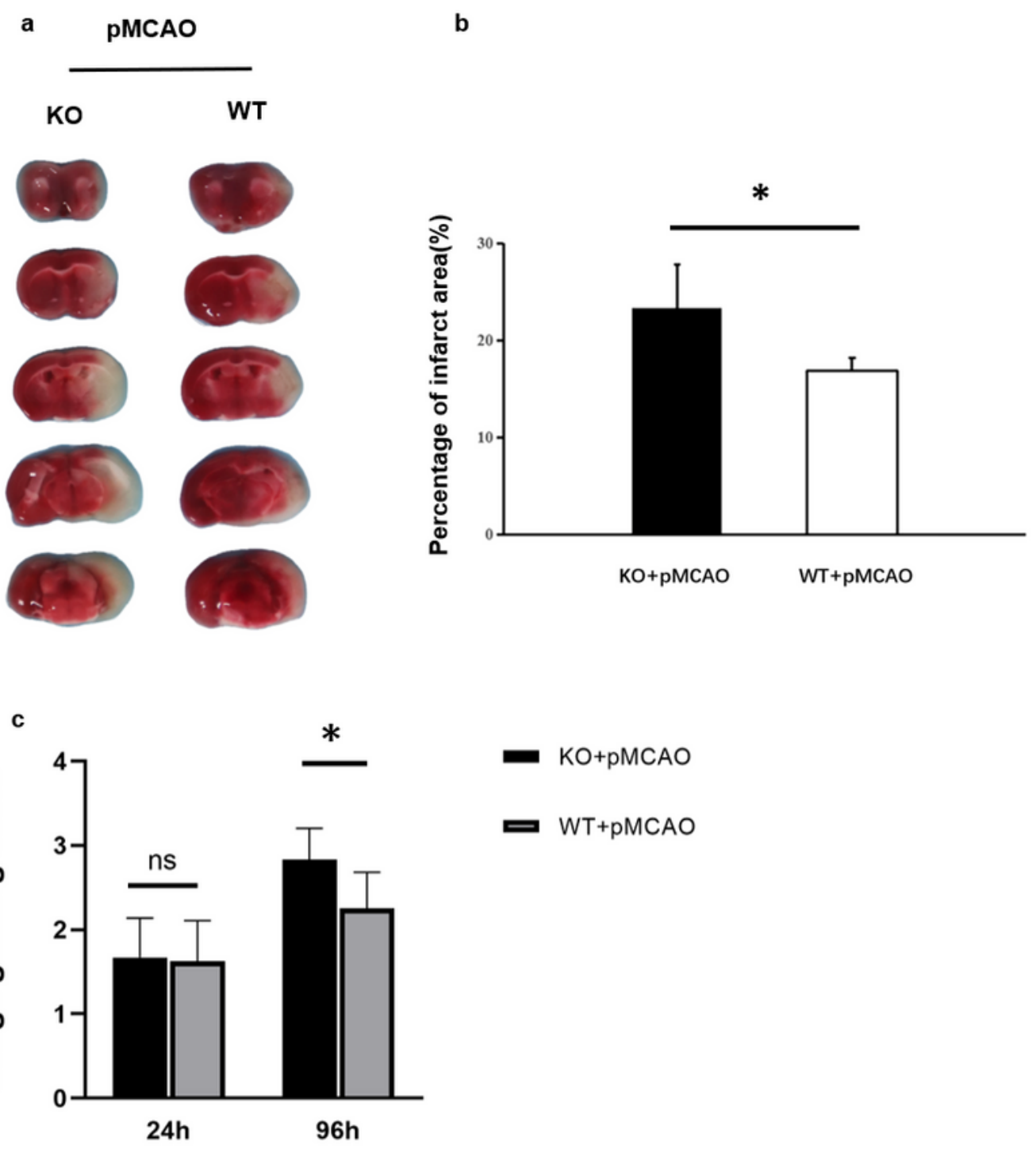

Figure 2 
(a) Representative TTC-stained coronal sections in each group after pMCAO. (b) Quantitative analysis of cerebral infarction volume corresponding to the TTC-stained sections ( $n=5$ per group). Quantification of infarct volume expressed as a percentage of hemispheric volume (\%) in each group. (c) Neurologic deficits were assessed at $24 \mathrm{~h}$ and $96 \mathrm{~h}$ after pMCAO ( $\mathrm{n}=10$ per group). Data are expressed as the means \pm SD. WT, wild-type; $K O$, knockout. ${ }^{*} \mathrm{P}<0.05$.

a

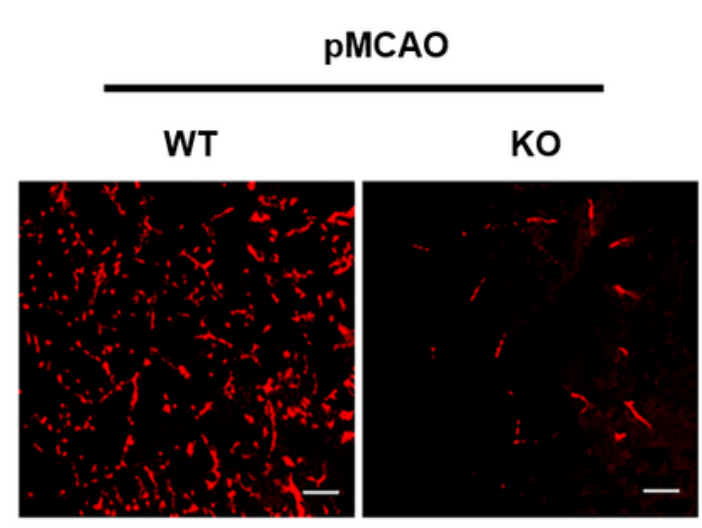

b

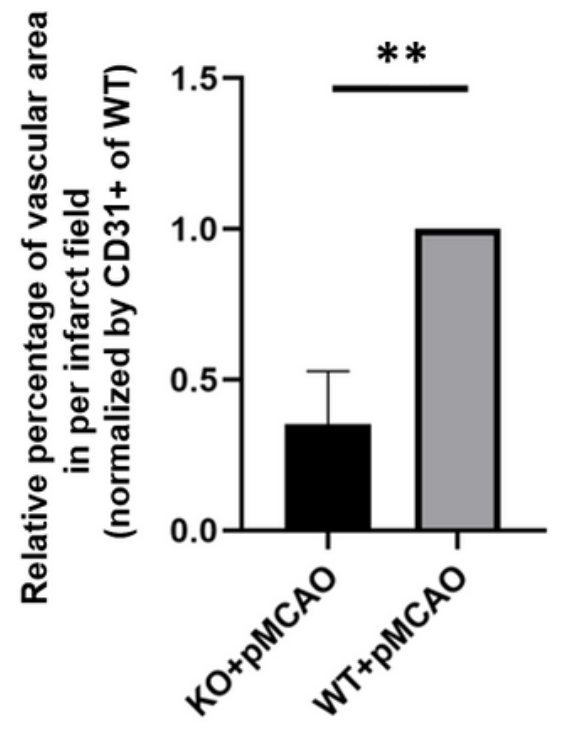




\section{Figure 3}

Angiogenesis was significantly decreased in Gucy1a3-KO mice following ischemic stroke

(a) Representative images of immunostaining of CD31 as a vascular density marker. Scale bar: $100 \mu \mathrm{m}$.

(b) Quantitative analysis showed that the vascular density was significantly decreased in the perifocal striatum of Gucy 1a3-KO mice ( $n=6$ per group). Data are expressed as the means $\pm S D$. ${ }^{\star *} P<0.01$ 
a

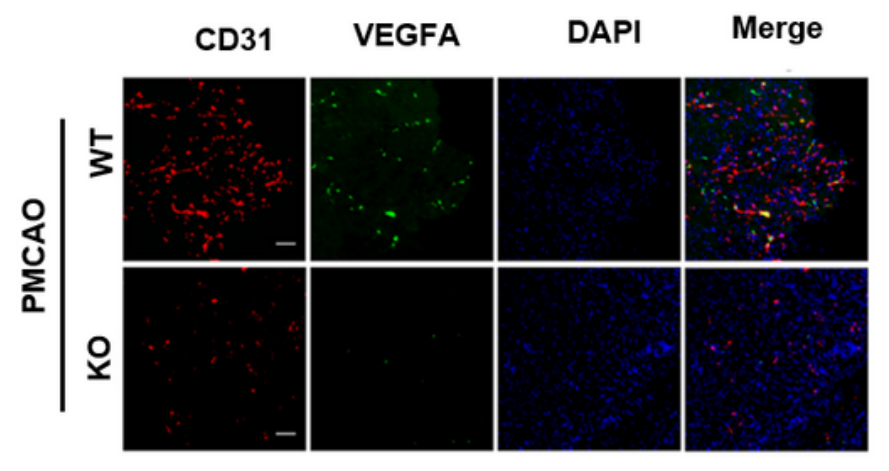

c

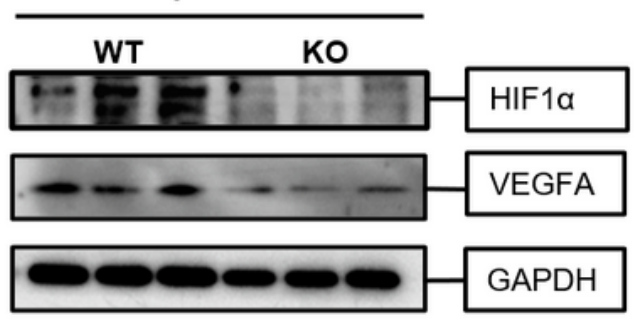

d

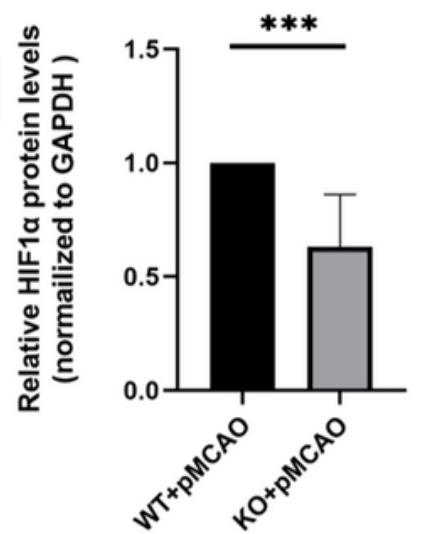

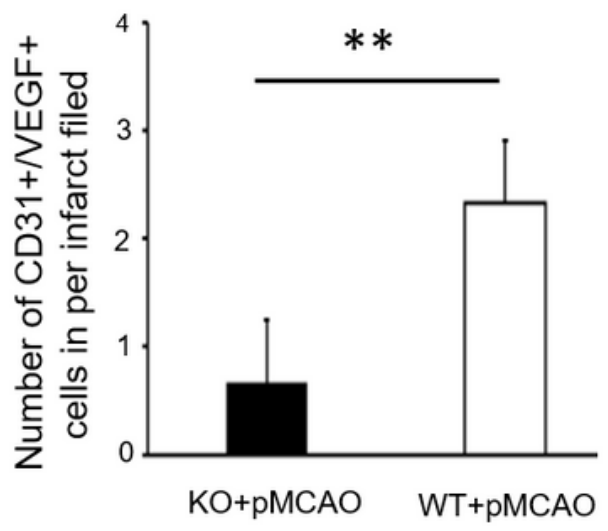

e

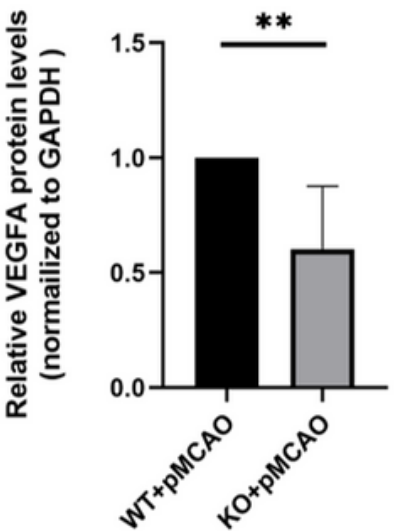

Figure 4

Gucy1a3 deletion decreases VEGFA and HIF-7a expressions in ischemic mice

(a) Immunofluorescence staining for VEGFA and CD31 with 4',6-diamidino-2-phenylindole in the perifocal striatum of Gucy1a3-KO and WT mice 96 hours after pMCAO. Scale bars: $100 \mu \mathrm{m}$. (b) Quantitative analysis of the number of CD $31^{+} /$VEGFA $^{+}$cells ( $n=6$ per group). (c) Western blotting of HIF- $1 \mathrm{a}$ and VEGFA 
proteins from ischemic brains. (d) Quantitative results of the HIF- 1 a to GAPDH bands ( $n=5$ per group). (e) Quantitative results of the HIF- 1 a to VEGFA bands ( $n=5$ per group). Data are expressed as the means \pm SD. ${ }^{*} \mathrm{P}<0.01$ and ${ }^{* * *} \mathrm{P}<0.001$.

\section{Supplementary Files}

This is a list of supplementary files associated with this preprint. Click to download.

- GUCY1A3KOmiceSupplementary.docx 